\title{
Qualitative investigation in explainable artificial intelligence: A bit more insight from social science
}

\author{
Adam Johs ${ }^{1}$, Denise Agosto ${ }^{1}$, and Rosina Weber ${ }^{1}$ \\ ${ }^{1}$ Drexel University College of Information Science and Technology
}

September 28, 2021

\begin{abstract}
We present a focused analysis of user studies in explainable artificial intelligence (XAI) entailing qualitative investigation. We draw on social science corpora to suggest ways for improving the rigor of studies where XAI researchers use observations, interviews, focus groups, and/or questionnaires to capture qualitative data. We contextualize the presentation of the XAI papers included in our analysis according to the components of rigor described in the qualitative research literature: 1) underlying theories or frameworks, 2) methodological approaches, 3) data collection methods, and 4) data analysis processes. The results of our analysis support calls from others in the XAI community advocating for collaboration with experts from social disciplines to bolster rigor and effectiveness in user studies.
\end{abstract}

\section{Hosted file}

Johs_et_al-Qual_Investigation_in_XAI.pdf available at https://authorea.com/users/438346/ articles/539573-qualitative-investigation-in-explainable-artificial-intelligence-a-bitmore-insight-from-social-science 